\title{
MedienPädagogik
}

Zeitschrift für Theorie und Praxis der Medienbildung

Jahrbuch Medienpädagogik 4.

Zweitveröffentlichung aus: Jahrbuch Medienpädagogik 4. (2005) Wiesbaden: VS Verlag für Sozialwissenschaften. Hrsg. v. Ben Bachmair, Peter Diepold und Claudia de Witt.

\section{Einleitung: Jahrbuch Medienpädagogik 4}

\author{
Ben Bachmair, Peter Diepold und Claudia de Witt
}

Ein Jahrbuch, herausgegeben von einer wissenschaftlichen Vereinigung, ist nicht primär auf ein Thema zugeschnitten. Es will vor allem den Stand der erziehungswissenschaftlichen Disziplin der Medienpädagogik erkenn- und diskutierbar machen. Die zentralen Eckpfeiler in diesem Jahrbuch Medienpädagogik 4 sind Mediendidaktik, Mediennutzung und Medienhandeln. Ein Teil der Texte speist sich aus der Herbsttagung der Kommission Medienpädagogik der Deutschen Gesellschaft für Erziehungswissenschaft von 2002 an der Humboldt Universität Berlin und der Frühjahrstagung 2003 an der Universität Erlangen-Nürnberg. Deren Themenschwerpunkte waren in Berlin „Empirische Ansätze und Theoriefragen zu neuen Medien” und in Nürnberg die Auseinandersetzung mit „medialen Räumen für Bildung”. Der andere Teil der Beiträge kommt ohne vorausgehende Diskussion in der Kommission Medienpädagogik aus den Arbeitsfeldern der Kolleginnen und Kollegen. Diese Beiträge beschäftigen sich mit Zukunftsfeldern der Medienpädagogik, diskutieren den Umgang mit digitalen Medien aus sozialwissenschaftlicher Perspektive oder stellen neue empirische Ansätze vor.

Blickt man ins politische Feld der Medienpädagogik, dann taucht ein völlig anderes Thema auf, das des Jugendmedienschutzes und seiner organisatorischen Neufassung durch den „Staatsvertrag über den Schutz der Menschenwürde und den Jugendschutz in Rundfunk und Telemedien”, der am 1. April 2003 in Kraft trat. Die Schubkraft für diese Veränderung lieferte die Amok-Tat eines Schülers in Erfurt, dessen Gewalttat Parallelen zur Spielesoftware vom Typ „Ego Shooter” aufwies. Auch Jahrbuch 4 geht nicht auf diese Thematik ein, die jedoch für die öffentliche Diskussion der Presse, ebenso für die Aktivitäten der Exekutive, wie für die Landesmedienanstalten im Vordergrund steht. Die institutionalisierte akademische Disziplin gewinnt ihre Themen offensichtlich in anderen Interessenzusammenhängen, Zwängen und Diskursen, als diejenigen, die für Ministerien oder die Landesmedienanstalten leitend sind. Für sie gibt es eher die journalistisch vermittelten Medien-Themen mit Nähe zur Skandalisierung, bei denen Medien als Auslöser oder Vorlagen für unerwünschtes Handeln der Kinder und Jugendlichen erscheinen. Zugleich wechseln die Themen, und zwar in der Logik der öffentlichen Berichterstattung, was nicht wenig dazu beiträgt, medienpädagogischen 
und mediendidaktischen Zielen und Innovationen den Charakter von Kampagnen aufzudrücken.

Wie wird die Medienpädagogik als akademische Disziplin damit umgehen? Holt sie sich Finanzierung und institutionelle Legitimation aus den Budgets und Zielen der Kampagnen wie Schule ans Netz ${ }^{1}$ oder „Schau hin”? Was „macht“ die Anbindung an oder die Distanz von Kampagnen mit den traditionellen Formen und Diskursen akademischer Forschung?

Zumindest liefern Innovationen wie der Jugendmedienschutz-Staatsvertrag Anlässe für die argumentative und systematisch theoretische Auseinandersetzung. So stellt der Jugendmedienschutz-Staatsvertrag den Gedanken der „Entwicklung von Kindern und Jugendlichen oder ihre Erziehung zu einer eigenverantwortlichen und gemeinschaftsfähigen Persönlichkeit” in den Mittelpunkt des juristischen Bemühens. Medienpädagogik könnte dies als Herausforderung betrachten, einen Bildungsbegriff oder vergleichbare Ideen in die akademische Diskussion einzubringen, denn diese Ausrichtung des Jugendmedienschutzes fußt auf einer langen Tradition pädagogischen Denkens mit deutlicher Nähe zum Konzept der Bildung. Gibt es in der Medienpädagogik nicht in Argumentationsdefizit, wenn es um die Anbindung oder die explizierte Distanz zur erziehungswissenschaftlichen Theorie und ihrer Tradition geht? Zwar hat sich die Medienpädagogik von der Medienwissenschaft einen textbezogenen Medienbegriff entliehen, der sich sehr wohl dazu eignet, argumentative und auch didaktische Verbindungen zwischen der Welt der Unterhaltungsmedien und der schulischen Buch-Welt zu schaffen. Ein aktualisiertes Bildungskonzept wäre jedoch eine lohnende Aufgabe, will sich die Disziplin nicht mit „Medienkompetenz” zufrieden geben. Eine, zwar auf den ersten und theoretischen Blick formelhaft leer erscheinende Ausrichtung auf die „,Entwicklung von Kindern und Jugendlichen oder ihre Erziehung zu einer eigenverantwortlichen und gemeinschaftsfähigen Persönlichkeit” beinhaltet die Chance, über die pädagogische Tradition eines Jean-Jacques Rousseau, der von der Natur des Menschen und deren Entfaltung durch Erziehung ausging, das Konzept der „Medienkompetenz” zu entfalten. (Siehe dazu u.a. die Beiträge von Meister/Hagedorn/Sander oder Treumann/Burkatzki/Strotmann/Wegener.) Dieter Baacke hat den Medienkompetenz-Begriff zwar für den Disput außerhalb der akademischen Disziplin der Medienpädagogik konkretisiert. Auch sind die Dimensionen von Medienkompetenz zur Verständigung mit der Politik usw. sicher hilfreich: Medienkritik (analytisch, reflexiv, ethisch); Medienkunde (informativ, instrumentellqualifikatorisch); Mediennutzung (rezeptiv-anwendend, interaktiv-handelnd); Mediengestaltung (in-

1 www.schulen-ans-netz.de

2 Zitat der Website http://www.schau-hin.info vom 15.10.03: „,SCHAU HIN! Die Aktion für mehr Erziehungsverantwortung im Umgang mit den elektronischen Medien. SCHAU HIN! gibt praxisnahe Hilfestellungen für den kindgerechten Umgang mit Medien, konkreten Rat und fundiertes Wissen von Experten an Eltern, Familien, Pädagogen weiter.“ Als „Partner“ werden genannt: HÖRZU, Bundesministerium für Familie, Senioren, Frauen und Jugend, ACOR, ARD, ZDF, intel. 
novativ, kreativ). Ohne erziehungswissenschaftlichen Bezugsrahmen lässt sich „Medienkompetenz” leicht didaktisch operationalisieren und in lehrbare Curricula gießen, wozu sich Beispiele anführen ließen. Besteht nicht doch die Gefahr, mit dem Instrument „Medienkompetenz” ein überprüfbares Lehrplangebilde zu schaffen, dem die Erziehungsidee abhanden gekommen ist; eine Erziehungsidee, die jedoch Noam Chomski mit seinen Überlegungen von Kompetenz und Performanz bei Sprache eröffnete und auf die sich Dieter Baacke ${ }^{3}$ selbstverständlich bezog? Um den Gedanken von Erziehung als Kompetenzentwicklung historisch zu verorten, eignet sich das folgende und im Rahmen der Medienpädagogik eher spröd antiquiert erscheinende Zitat von Jean-Jacques Rousseau ${ }^{4}$ :

„Erziehung kommt uns von der Natur oder den Menschen oder den Dingen. Die innere Entwicklung unserer Fähigkeiten und unserer Organe ist die Erziehung durch die Natur. Den Gebrauch, den man uns von dieser Entwicklung zu machen lehrt, ist die Erziehung durch die Menschen, und der Gewinn unserer eigenen Erfahrungen mit den Gegenständen, die uns affizieren, ist die Erziehung durch die Dinge” (S. 109).

Im Kern geht es um Entwicklung des Kindes als Entfaltung und Entäußerung seiner in ihm angelegten Möglichkeiten, wozu sehr wohl die soziale, dingliche und kulturelle Umwelt von zentraler Bedeutung ist. Die didaktische Idee, etwas in die Menschen hineinzuverlagern, als Aneignung im Sinne von Belehrt werden, von Pauken, einen Lehrplan zu übernehmen, dies ist jedoch Rousseaus Erziehungsidee fremd. Dagegen passt hierzu der Gedanke der Kompetenz, deren Förderung durch die kulturelle und soziale Umwelt geschieht. Also, auch der Gedanke der Medienkompetenz, dieser jedoch nicht in der Variante der Vermittlung von Medienkritik, Medienkunde, Mediennutzung und Mediengestaltung, enthält das erziehungswissenschaftliche Potential, das eben auch dem kodifizierten Jugendmedienschutz inhärent ist, die Beziehung sich entfaltender Subjekte in einer von Medien geprägten Kultur zu thematisieren. Das zentrale pädagogische Motiv des Jugendmedienschutzes mit der Ausrichtung auf die „Entwicklung von Kindern und Jugendlichen oder ihre Erziehung zu einer eigenverantwortlichen und gemeinschaftsfähigen Persönlichkeit” ist jedoch in Rousseaus Denkansatz verortet, auch wenn dieser konzeptionelle Rahmen von Medienkompetenzförderung als Förderung der Entäußerung in sozialen und kulturellen Kontexten vermutlich verloren gegangen ist. Die Diskutanten zum Jugendmedienschutz sind an diese Tradition zu erinnern.

Beiträge in diesem Band greifen Aspekte dieser Diskussion um Erziehung als Entwicklung im Prozess der subjektiven Aneignung und Entäußerung in dem spezifischen kulturellen Kontext didaktisch organisierter Situa-

3 Baacke, Dieter: Kommunikation und Kompetenz. Grundlegung einer Didaktik der Kommunikation und ihrer Medien. München 1980. 3. Auflage, 1. Auflage 1973.

4 Rousseau, Jean-Jacques: Emile oder über die Erziehung. Stuttgart 1963, Nachdruck 2001 (Original 1760). 
tionen auf, beispielsweise der Beitrag von Willett, der von Meister/Hagedorn/Sander, der von Grabowski/Krauß, auch der von Preußler/Schulz-Zander.

Damit scheint eine Bildungsdiskussion auf, die sicher weitere Facetten braucht, z.B. die, die Dietrich Benner ${ }^{5}$ mit Hilfe von Wilhelm von Humboldts Bildungstheorie festmacht, indem er auf die „Weltinhalte” (S. 20) eingeht und nach deren Funktion für Bildung fragt. Dazu gehören heute Medien, gerade auch diejenigen, deren Erscheinungsform und Funktion mutieren, indem sie zu Repräsentationsmodi von Texten in aktuellen Arrangements mit Events werden. (Konkret geht es um Formate wie „Deutschland sucht den Superstar”.) Zu fragen bleibt, wie hier die Beziehung von Subjekten und Erziehung sowie die von „Menschheit” und Kultur medienpädagogisch, bildungstheoretisch zu bedenken ist. Reicht Erziehung als Entfaltung? Reicht es, in diesem Sinne über die Entfaltung von Medienkompetenz praktisch nachzudenken? Welche Rolle spielen „Medien” in diesem Entfaltungsprozess als Kultur? Welche reflexiven Prozesse der Selbstvergewisserung der Menschen in dieser medial geprägten Kultur korrelieren mit welchen Formen von Reflexivität, die in den aktuellen Medien- und Ereignisarrangements angelegt sind?

In einem ersten Teil des Jahrbuchs werden (medien-)didaktische Überlegungen für die Gestaltung medialer Räume für Bildungs- und Lernziele angestellt. So untersucht Bardo Herzig u.a. anhand des Fernstudiengangs Medien (FESTUM) die Frage, welchen Stellenwert der Allgemeinen Didaktik im Kontext von Lehren und Lernen in virtuellen Räumen zukommt. Ausgehend von einer Präsenzveranstaltung skizziert er entsprechende Lehr- und Lernprozesse. In der anschließenden Diskussion werden Veränderungen und didaktische Konsequenzen genannt, die sich beim Wechsel von realen in virtuelle Räume ergeben. Manche Lernmethoden eignen sich besser als andere für den Einsatz in virtuellen Räumen, so z.B. die offene Lernumgebung. Rolf Schulmeister beschreibt in zwei anschaulichen Beispielen zum Erlernen der Gebärdensprache die Vorteile solcher Lernumgebungen, nämlich die Möglichkeiten zu hohen Graden der Aktivität und zur intensiven Kommunikation. Hugo Kremer und Franz Gramlinger befassen sich in ihrem Beitrag mit der Konzeption, Vorbereitung und Durchführung von virtuellen Konferenzen. Es werden die Potentiale dieses „Instruments der Hochschuldidaktik“ diskutiert, aber auch die Grenzen der Realisierung in der universitären Lehre aufgezeigt. Marco Kalz, Jörg Stratmann und Michael Kerres gehen den Fragen nach, wie eine didaktische Innovation in einer Notebook-Universität aussehen kann und in welchen Szenarien eine sinnvolle Verwendung der Endgeräte stattfindet. Sie stellen dafür ein Rahmenmodell einer Notebook-Universität über die Ebenen Individuum, Gruppe und Organisation vor. Der eCampus Duisburg ist dabei Hauptgegenstand ihrer Überlegungen. Einen Einblick in

5 Benner, Dietrich: Wilhelm von Humboldts Bildungstheorie. Eine problemgeschichtliche Studie zum Begründungszusammenhang neuzeitlicher Bildungsreform. Weinheim, München 2. Auflage 1995. 
die Konzeption einer Computer-Studienwerkstatt an der TU Darmstadt gibt Michael Diéz Aguilar. Diese reicht von der Raumgestaltung bis zu einem permanenten, selbstreflexiven Projekt von Studierenden und Lehrenden. Aguilar beschreibt die Leitideen, informationspädagogischen Rahmenbedingungen und Gestaltung dieses „Bildungsraums“.

Im zweiten Teil geht es um zeitgemäße Sichtweisen auf Medienwissen und Medienhandeln. Hans-Dieter Kübler stellt die gegenwärtige Normen- und Kanondiskussion in Frage und kritisiert, dass einer Medienwirklichkeit immer noch eine wirkliche empirische Realität gegenübergestellt wird. Er plädiert dafür, dass eine pädagogisch orientierte Wissensforschung stärker in der Medienpädagogik Eingang finden muss und diese damit ihr Profil erweitere. Auf diesem Wege können Weltwissen und Medienwissen in ein Wechselverhältnis gebracht werden. Rebekah Willett geht davon aus, dass durch den alltäglichen Umgang mit den informellen Settings digitaler Kulturen lernende Erfahrungen bei Heranwachsenden im Alter von 9 bis 13 Jahren entstehen. In spielerischen Interaktionen eignen sich Jugendliche Inhalte und Lernstile an. Sie präsentiert dazu Daten eines Projektes, das dieses Lernen von Jugendlichen in digitalen Kulturen untersucht und geht der Frage nach, welche Software für Jugendliche geeignet ist. Um ein möglichst umfassendes Bild über das Medienhandeln Jugendlicher im Alter von 12 bis 20 Jahren zu gewinnen, setzen Klaus Peter Treumann, Eckhard Burkatzki, Mareike Strotmann und Claudia Wegener das Bielefelder Medienkompetenz-Modell und das multivariate Verfahren der Hauptkomponentenanalyse ein. Mit dessen Hilfe ist es möglich, die Hauptquellen der Variationen bzw. der Unterschiede zu rekonstruieren, welche die Jugendlichen in ihren Medienaktivitäten zeigen. Das Phänomen Medienkompetenz bei Jugendlichen untersuchen auch Dorothee M. Meister, Jörg Hagedorn und Uwe Sander. Dazu operationalisieren sie zunächst mit Hilfe von quantitativen Befragungen die Dimensionen der Medienkompetenz und konkretisieren diese in qualitativen Befragungen. Die Entwicklung von Medienkritik wird anhand einer exemplarischen Fallrekonstruktion dargestellt. Aus einer sozialwissenschaftlichen Perspektive betrachten Jana Dittmann und Winfried Marotzki das Vetrauensphänomen in digitalen Räumen. Am Beispiel von Online-Auktionen geben sie Einblicke in die Diskussion über Vertrauenskulturen und entwickeln Perspektiven für die Vertrauenskultur virtueller Communities.

Welche Veränderungen bewirken die Informations- und Kommunikationstechnologien in Schulen tatsächlich? Inwieweit verändern sich Lehr- und Lernprozesse? Diesen und vielen anderen Fragen gehen in einem dritten Teil Annabell Preussler und Renate Schulz-Zander nach, indem sie die Ergebnisse der SITE- und SelMa Studien unter dem Aspekt selbstregulierten und kooperativen Lernens vorstellen und diskutieren. Sigrid Blömeke, Christiane Müller und Dana Eichler stellen hier ein Projekt zur empirischen Unterrichtsforschung vor. Mit Hilfe von Videoaufnahmen sollen Handlungsmuster von Lehrpersonen beim Einsatz neuer Medien identifiziert und anschließend in einen Bezug zu Fach und Expertisegrad gebracht werden. Ziel ist u.a. die 
Weiterentwicklung von Lehrerhandeln beim Einsatz neuer Medien im Unterricht. ELearning ist auf eine hohe Handlungskompetenz der Lernenden angewiesen. Besonders die Fähigkeit, Informationen zu recherchieren, zu evaluieren und zu reduzieren stellt eine wichtige Anforderung dar. In der von Udo Hinze und Gerold Blakowski vorgestellten Fallstudie wird aufgezeigt, wie sich unterschiedliche Informationskompetenzen auf Lernerfolg, Lernzufriedenheit und Nutzungsmodi der Medien auswirkt. Susanne Grabowski und Matthias Krauß befassen sich hier mit der Frage, was Computerkunst überhaupt ist und welche Prinzipien sie hat. Der Streifzug durch die Computerkunst beginnt bei Vilém Flusser, der uns technische Bilder näher bringt, und führt über Vorschläge zur Gestaltung von digitalen Medien für Lernumgebungen bis hin zu einem praktischen Beispiel aus dem Forschungsprojekt COMPART. Digitale Medien sind nach Richard Stang ein wichtiger Motor dafür, dass sich unsere Gesellschaft stetig zur „Wissensgesellschaft" weiterentwickelt. Ihn interessiert deshalb, welche Auswirkungen die neuen Medien insbesondre auf die organisationale Struktur von traditionellen Bildungsinstituten haben. Er liefert mit seiner vorgestellten Untersuchung einen Beitrag zur Organisationsforschung in der Weiterbildung.

In dem vierten Teil des Jahrbuchs wird explizit die Mediennutzung an Bildungsportalen als Medien der Wissensaneignung analysiert und die Erfolgsfaktoren herausgestellt. Denn mittlerweile hat sich der Deutsche Bildungsserver zu einem stark benutzten und inhaltlich hochwertigen Internetportal entwickelt. Um eine angemessene Weiterentwicklung zu gewährleisten ist es wichtig zu wissen, wer die Nutzer sind und wie die Informationen angenommen und bewertet werden. Elke Brenstein und Olaf Kos stellen hier die Ergebnisse von Online-Befragungen und Fokusgruppenuntersuchungen zu inhaltlichen, strukturellen und grafischen Aspekten der Gebrauchstauglichkeit vor und geben Empfehlungen zur Optimierung der Angebote. Ziel der Studie von Michael Kluck und Susanne Politt ist die qualitative Untersuchung des Suchverhalten und typischer Suchanfragen von Normalnutzern von Bildungsportalen. Grundlage dafür waren Logfiles des Deutschen Bildungsservers von Oktober 2001 bis April 2002. Abschließend machen sie Vorschläge für die Weiterentwicklung von Bildungsportalen. Christian Swertz ist an der pädagogischen Konzeption der Online-Bildungsplattform für die Bundesinitiative „Jugend ans Netz“ (BIJaN) beteiligt und entwickelt dazu einen Ansatz der Mediengestaltung. Vor dem Hintergrund medienpädagogischer, didaktischer und bildungstheoretischer Ansätze skizziert er die didaktische Konzeption und stellt das entwickelte Metadatensystem sowie Ansätze der technischen Umsetzung vor. 